\title{
Early versus late introduction of stimuli in psychophysical discriminations by pigeons ${ }^{1}$
}

\author{
THOMAS L. MENTZER \\ NEWCOMB COLLEGE, TULANE UNIVERSITY
}

\begin{abstract}
Brightness difference thresholds were obtained from pigeons using a discrete-trial conditioning method. Three Ss were pretrained in the absence of the discriminative stimulus to see whether delayed exposure to difficult discriminations results in good stimulus control. The thresholds obtained were higher and more variable than those of three Ss pretrained with the discriminative stimulus present. The poor stimulus control of the delayed-discrimination $S s$ was attributed to competition from position habits which carried over from prediscrimination into discrimination training.
\end{abstract}

In previous work (Mentzer, 1966), brightness difference thresholds were obtained from pigeons by a discrete-trial conditioning method. The thresholds varied markedly between Ss. Poor stimulus control was attributed to aversive properties of the difficult discriminations. The Ss stopped responding when first confronted with the difficult discriminations during pretraining, and starvation was required to force them to respond. In this experiment, pigeons were pretrained in the absence of discriminative stimuli in order to determine whether postponing the exposure to difficult discriminations results in lower, more stable thresholds.

Subjects

The Ss were six naive male White Carneaux pigeons over five years of age. The Ss were maintained at $75 \%$ of their free-feeding body weights. Water and grit were always available in the home cages.

\section{Apparatus}

Training took place in a two-key operant conditioning space equipped with a Gerbrands food hopper, white noise speaker, and ventilating fan. A dual-path optical system containing a tungsten filament lamp, relay-driven shutters, and a motor-driven Kodak neutral density filter tablet provided the stimuli. Method

The procedure is described in detail in an earlier study (Mentzer, 1966). Two keys $3 / 4$ in. in diameter were illuminated equally by white light. At the start of a trial, a spot of white light $3 / 16$ in. in diameter appeared on one of the keys, superimposed over the background, and a buzzer sounded. A response on the spot key was reinforced by $3.4 \mathrm{sec}$ access to food. A response to the other, incorrect key was not rewarded. Any response ended the trial, terminating the spot and buzzer, and started a 15-sec intertrial interval (ITI) during which only the background light was present. Any response occurring during the ITI was punished by a 10-sec delay of trial onset. Ten spot intensities .02 to $2.49 \mathrm{~mL}$ brighter than the $.61 \mathrm{~mL}$ background were presented. The spot appeared on each key and at each intensity equally often in a 60-trial daily experiment. Incorrect responses caused the stimulus conditions to remain unchanging on succeeding trials until $S$ pecked the other, correct key. Each correction response and all of the successive incorrect responses preceding it were counted as a single error trial in computing the psychophysical functions and counted as only one of the 60 daily trials. The difference threshold was that stimulus intensity to which $75 \%$ correct responses were made in a five-day block. When the $75 \%$ value fell between two intensities, the threshold was estimated by linear interpolation.

During pretraining, three control (C) group Ss were trained to peck the right-hand key for continuous reinforcement. The brightest spot was present on that key, and the background intensity was reduced to $0.61 \mathrm{~mL}$. The $\mathrm{Ss}$ were then trained to track the spot as it moved randomly from key to key for at least 250 reinforcements. Next, the ITI was introduced following each trial. After a minimum of $\mathbf{1 2 0}$ trials, the spot intensity was varied from trial to trial. When near perfect discrimination had been achieved, the background illumination was increased to $.61 \mathrm{~mL}$ and data collection was begun.

An experimental group (E) of three Ss was matched according to weight to members of Group $C$. The Group E Ss were treated as much like their Group C partners as possible with respect to training durations at each stage. However, for Group E, the stimulus spot was blocked out until each S's Group C partner had reached the data collection stage. Reinforcement was programmed in exactly the same way for the two groups, but the discriminative stimulus was not available to guide the choices of Group E during pretraining. During the data collection stage, the experimental conditions were identical for the two groups.

\section{Results and Discussion}

Figure 1 shows the Weber fractions for successive blocks of five days for the matched pairs of Ss. There is no overlap within any of the pairs, and only twice did a Group $E$ bird make a threshold lower than the highest made by a Group $\mathrm{C}$ bird. Only one Group $E$ bird, 12-E, showed any sign that it might attain the degree of stimulus control achieved by Group $\mathrm{C}$ 


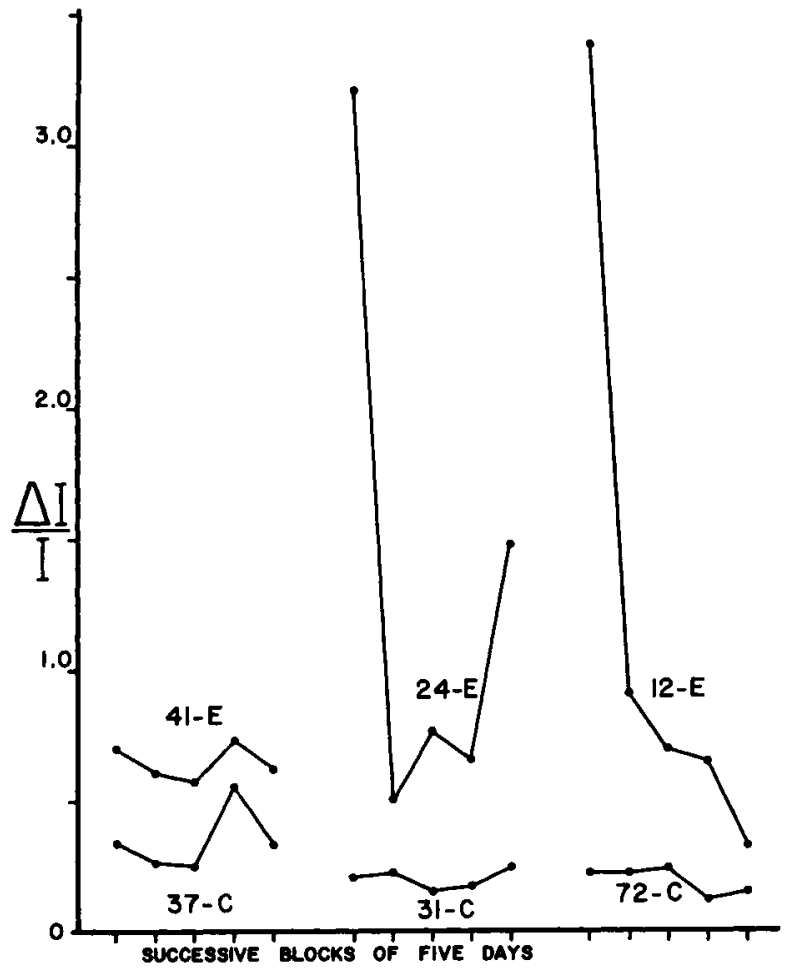

Fig. 1. Weber fractions for successive five-day blocks for matched pairs of Ss.

if training were extended. The lowest thresholds of Ss 31-C and 72-C are of the same order as those reported for comparable species (Mead, 1939).

One might have predicted beforehand that Group $\mathrm{E}$ would perform as well or perhaps better than Group C. During pretraining Group $E$ was permitted to learn the subsidiary tasks, i.e., responding to two keys and the ITI discrimination, without simultaneously having to acquire the observing response. Also, the low reinforcement rate experienced during pretraining was replaced by a higher payoff rate as the Group E birds learned to attend to the cue stimulus after pretraining. Birds 24-E, 12-E, and 41-E were rewarded for $44 \%, 45 \%$, and $45 \%$, respectively, of their trial responses during pretraining and for $61 \%$.
$61 \%$, and $63 \%$, respectively, of all trial responses in their best five-day blocks after pretraining.

Group $\mathrm{C}$, on the other hand, experienced a decrease in reinforcement rate when the difficult discriminations were introduced. Birds 37-C, 31-C, and $72-C$ were rewarded for $71 \%, 66 \%$, and $76 \%$, respectively, of all trial responses during their best five-day blocks after receiving nearly $100 \%$ reinforcement during pretraining.

Terrace (1963) has shown that errors are detrimental to the acquisition of stimulus control. Pigeons trained to discriminate with errors often form position preferences, exhibit avoidance behaviors in the presence of the negative stimulus, and generally perform more poorly than Ss trained to discriminate without errors. On this basis the high error rate experienced by the Group $E$ Ss during pretraining might be expected to result in the formation of position habits which would interfere with the acquisition of stimulus control behavior during training. Maier (1949) found that rats faced with insoluble discriminations formed "fixated" position habits which persisted even after the discriminations were again made soluble. Ss $24-\mathrm{E}, 12-\mathrm{E}$, and $41-\mathrm{E}$ made $74 \%, 60 \%$, and $55 \%$, respectively, of all pretraining responses on the left key. Although the direction of the preferences did sometimes change, the Group $\mathrm{E}$ Ss also tended to maintain stronger position habits after pretraining than did Group $C$.

It was concluded that delayed exposure to difficult discriminations does not result in good stimulus control if the prediscrimination period is accompanied by a low reinforcement rate.

\section{References}

MAIER, N. R. F. Frustration. New York: McGraw-Hill, 1949.

MEAD, L. C. Thresholds of visual intensity discrimination in phylogeny. Amer. J. Psychol., 1939, 52, 465-467.

MENTZER, T. L. Comparison of three methods for obtaining psychophysical thresholds from pigeons. J. comp. physiol. Psychol, 1966, 61, 96-101.

TERRACE, H. S. Discrimination learning with and without "errors." J. exp. Anal. Behav., 1963, 6, 1-27.

Note

1. This research was supported by Public Health Service Grant MH 08575-01. 